OPEN ACCESS

Edited by:

Chengqi $X u$,

Huazhong University of Science and

Technology, China

Reviewed by:

Ningjian Wang,

Shanghai Jiao Tong University, China

Yan Bi,

Nanjing Drum Tower Hospital, China

Yan Yang,

Huazhong University of Science and

Technology, China

*Correspondence:

Yuping Sun

544481723@qq.com

Cheng Hu

alfredhc@sjtu.edu.cn

Specialty section:

This article was submitted to

Cellular Endocrinology,

a section of the journal

Frontiers in Endocrinology

Received: 25 January 2022

Accepted: 09 February 2022

Published: 02 March 2022

Citation:

Kahaer M, Zhang B, Chen W, Liang M,

He Y, Chen M, Li R, Tian T, Hu C and

Sun Y (2022) Triglyceride Glucose Index Is More Closely Related to

Hyperuricemia Than Obesity

Indices in the Medical Checkup

Population in Xinjiang, China.

Front. Endocrinol. 13:861760.

doi: 10.3389/fendo.2022.861760

\section{Triglyceride Glucose Index Is More Closely Related to Hyperuricemia Than Obesity Indices in the Medical Checkup Population in Xinjiang, China}

\author{
Mayina Kahaer 1,2, Bei Zhang ${ }^{2}$, Wujin Chen ${ }^{2}$, Meiting Liang ${ }^{2}$, Yi He ${ }^{2}$, Miao Chen ${ }^{3}$, Rui $\mathrm{Li}^{2}$, \\ Tingting Tian ${ }^{2}$, Cheng $\mathrm{Hu}^{4,5^{*}}$ and Yuping Sun ${ }^{2 *}$ \\ ${ }^{1}$ School of Public Health, Xinjiang Medical University, Urumqi, China, ${ }^{2}$ Department of Microbiology, School of Basic Medical \\ Sciences, Xinjiang Medical University, Urumqi, China, ${ }^{3}$ Department of Rheumatology and Immunology, Shanghai Jiao Tong \\ University Affiliated Sixth People's Hospital, Shanghai, China, ${ }^{4}$ Shanghai Key Laboratory of Diabetes Mellitus, Shanghai \\ Clinical Center for Diabetes, Shanghai Diabetes Institute, Shanghai Jiao Tong University Affiliated Sixth People's Hospital, \\ Shanghai, China, ${ }^{5}$ Department of Endocrinology and Metabolism, Fengxian Central Hospital Affiliated to the Southern \\ Medical University, Shanghai, China
}

Background: Hyperuricemia (HUA) is a metabolic anomaly with an increased incidence rate, causing a global medical burden. Several studies have confirmed that obesity and insulin resistance (IR) are the risk factors for HUA. Reports on the predictive power of different obesity indices for HUA are limited. This study aimed to compare the association between different general, abdominal, and visceral obesity indices and markers of the IRtriglyceride glucose (TyG) index with serum uric acid (SUA) and to assess the ability of these indices to predict HUA.

Methods: A total of 2243 participants were recruited from Barkol County Hospital and surrounding township hospitals in Xinjiang. Obesity indices, including the atherogenic index of plasma, cardiometabolic index, visceral adiposity index, lipid accumulation product index, a body shape index, body roundness index, waist circumference, waistto-height ratio, body mass index, and TyG index, were divided into four quartiles. Moreover, partial correlations and logistic regression were used to analyze the association between these indices and SUA. The area under the curve (AUC) and receiver operating characteristic curves were used to analyze the predictive value of these indices for HUA.

Results: After controlling for confounding variables, the association between the TyG index and HUA was stronger than that between the obesity indices in both males and females. The odds ratios (ORs) for HUA in the highest quartile of the TyG index were 2.098 (95\% confidence interval, 1.555-2.831) in males and 7.788 (95\% Cl, 3.581-16.937) in females. For males, the AIP, CMI, VAl, LAP index, and TyG index were able to discriminate HUA, and the TyG index showed the highest AUC value of $0.586(95 \% \mathrm{Cl}, 0.557-0.614$; $P<0.001)$. For females, all indices, except BMI, can discriminate HUA. Moreover, the 
visceral obesity index CMl showed the highest AUC value of 0.737 (95\% Cl, 0.691-0.782; $P<0.001)$. Meanwhile, the TyG index had a relatively high AUC value of $0.728(95 \% \mathrm{Cl}$, 0.682-0.773; $P<0.001)$.

Conclusion: The TyG index was significantly related to HUA and was superior to obesity indices in identifying HUA in the medical checkup population in Xinjiang, China.

Keywords: Triglyceride glucose index, serum uric acid, obesity indices, hyperuricemia, medical checkup population

\section{INTRODUCTION}

Uric acid is the final product of purine metabolism, and hyperuricemia (HUA) is caused by the excessive production or insufficient excretion of uric acid in the body, the incidence rate of which is increasing annually worldwide. Data from a national survey have demonstrated that the prevalence of HUA among Chinese adults during 2010-2014 was $13.3 \%$ (1). According to the National Health and Nutrition Examination Survey, approximately $21 \%$ of American adults have HUA (2). The age-standardized prevalence of HUA in the general Korean population is $11.4 \%$ (3). Simultaneously, a number of epidemiological studies have reported that HUA causes gout and increases the risk of ischemic stroke, acute myocardial infarction, and other cardiovascular events $(4,5)$.

Both obesity and insulin resistance (IR) are associated with HUA $(6,7)$. The general obesity index body mass index (BMI) and abdominal obesity indices waist circumference (WC) and waist-to-height ratio (WHtR) have some implications in predicting the incidence of HUA. However, these indices cannot clearly distinguish between visceral and subcutaneous fats. Previous studies have confirmed a positive correlation between visceral fat deposition and increased uric acid production (8). Since the direct estimation of visceral fat requires diagnostic imaging, which is expensive and has low epidemiological availability, there has been increasing interest in finding simple and effective alternative markers of visceral obesity. In recent years, some lipid and visceral obesity-related indices, such as visceral adiposity index (VAI), lipid accumulation product (LAP) index, atherogenic index of plasma (AIP), cardiometabolic index (CMI), a body shape index (ABSI), and body roundness index (BRI), have also been proposed as supplementary indices to assess obesity and to predict the incidence of related metabolic diseases. The triglyceride glucose (TYG) index is a marker of IR and is associated with uric acid through obesity (9). A comparison with the gold standard method revealed that the TyG index was more suitable for the determination of IR than alternative indices, such as the homeostasis model assessment-estimated insulin resistance.

Many studies have investigated these indices in relation to cardiovascular diseases and diabetes, but few have predicted HUA (10, 11). Simultaneously, although many studies have pointed out that obesity indices can predict the risk of HUA, there was no conclusion on which indices were more suitable for predicting the risk of HUA in Xinjiang population, China.
Therefore, this study aimed to use a cross-sectional survey to analyze and compare six visceral obesity indices (AIP, CMI, VAI, LAP index, ABSI, and BRI) with the general obesity index BMI, abdominal obesity indices $\mathrm{WC}$ and WHtR, and TyG index to predict the risk of HUA, determine more suitable risk predictors for HUA in our population, and provide a basis for early prevention of HUA.

\section{METHODS}

\section{Study Participants}

This was a cross-sectional, observational study. All participants were selected from the medical checkup population of Barkol County Hospital and the surrounding township hospitals from May 2016 to May 2021. A total of 2243 participants (age range, 20-68 years) were included in this study. Individuals with any of the following conditions were excluded: (1) chronic kidney disease or renal function impairment, (2) long-term use of uric acid-lowering drugs, (3) malignant tumors, and (4) autoimmune diseases that may affect SUA levels. Trained research interviewers administered standardized questionnaires through face-to-face interviews. The questionnaire included age, sex, medication history, and medical history. Height, weight, and WC were measured using standard methods. Blood pressure was measured using an electronic sphygmomanometer. Fasting blood samples $(5 \mathrm{~mL})$ were drawn from the participants under strict aseptic conditions and the serum was separated by centrifugation at $3000 \mathrm{rpm}$ for $15 \mathrm{~min}$ after clotting. Myriad BS-800M fully automated biochemical analyzer and its matching reagents were used to assess the level of serum uric acid (SUA), fasting plasma glucose(FPG), high-density lipoprotein cholesterol (HDL-C), low-density lipoprotein cholesterol (LDLC), total cholesterol (TC), triacylglycerol (TG), blood urea nitrogen (BUN), and creatinine (Cre) in Barkol County Hospital.

\section{Definition and Obesity Index Calculations}

HUA was defined as an SUA level $>7.0 \mathrm{mg} / \mathrm{dL}$ according to the guidelines for the diagnosis and management of HUA and gout in China (2019) (12). According to previous studies, obesity indices were calculated using the following formula $(13,14)$ :

$$
\begin{gathered}
\text { BMI = weight }(\mathrm{kg}) / \operatorname{height}^{2}\left(\mathrm{~m}^{2}\right) \\
\text { AIP }=\log (\mathrm{TG}[\mathrm{mmol} / \mathrm{L}] / \mathrm{HDL}-\mathrm{C}[\mathrm{mmol} / \mathrm{L}])
\end{gathered}
$$




$$
\begin{aligned}
& \mathrm{CMI}=\mathrm{TG} / \mathrm{HDL}-\mathrm{C} \times \mathrm{WHtR} \\
& \operatorname{VAI}(\text { males })=(\mathrm{WC}[\mathrm{cm}] / 39.68+ \\
& 1.88 \times \mathrm{BMI}]) \times(\mathrm{TG}[\mathrm{mmol} / \mathrm{L}] / 1.03) \\
& \times(1.31 / \mathrm{HDL}-\mathrm{C}[\mathrm{mmol} / \mathrm{L}]) \mathrm{VAI} \text { (females) } \\
& =\left(\mathrm{WC}[\mathrm{cm}] / 36.58+\left[\begin{array}{r}
1.89 \times \mathrm{BMI}]) \times(\mathrm{TG}[\mathrm{mmol} / \mathrm{L}] / 0.81) \\
\times(1.52 / \mathrm{HDL}-\mathrm{C}[\mathrm{mmol} / \mathrm{L}])
\end{array}\right.\right. \\
& \text { LAP index }(\text { females })=\mathrm{TG}(\mathrm{mmol} / \mathrm{L}) \times(\mathrm{WC}[\mathrm{cm}]-58) \\
& \text { LAP index }(\text { males })=\mathrm{TG}(\mathrm{mmol} / \mathrm{L}) \times(\mathrm{WC}[\mathrm{cm}]-65) \\
& \text { TyG index }=\log (\mathrm{TG}[\mathrm{mmol} / \mathrm{L}] \\
& \times \text { fasting plasma glucose }(\mathrm{FPG})[\mathrm{mmol} / \mathrm{L}] / 2) \\
& \mathrm{ABSI}=\mathrm{WC}(\mathrm{cm}) /(\text { height }[\mathrm{cm}])^{1 / 2} \times\left(\mathrm{BMI}^{2}\right)^{1 / 3} \\
& \left.\mathrm{BRI}=364.2-365.5 \times[1-(\mathrm{WC} / 2 \pi) /(0.5 \times \text { height })]^{2}\right]^{1 / 2}
\end{aligned}
$$

\section{Statistical Analyses}

The measures of normal distribution are expressed as means \pm standard deviation, and the two groups were compared using an independent sample t-test. One-way analysis of variance was used to compare more than two groups. Non-normally distributed measures are expressed as M (P25, P75), the Mann-Whitney U-test was used for comparison between two groups, and the Kruskal-Wallis H-test was used for comparison between more than two groups. Partial correlation analysis was performed to assess the correlation between the different indices and SUA. Logistic analysis was performed to analyze the association between the different indices and HUA. A receiver operating characteristic (ROC) curve was used to analyze the predictive value of the different indices for HUA. All statistical analyses were performed using the Statistical Package for the Social Sciences version 21.0 (International Business Machines Corporation) and GraphPad Prism version 6.0. Statistical significance was defined as a two-tailed $P$-value $<0.05$.

\section{RESULTS}

A total of 2243 participants (1616 males and 627 females) were enrolled in the study, with an age range of 20-69 years (mean age, $41.55 \pm 12.70$ years). The basic characteristics of all participants are summarized in Table 1. Participants with HUA were older than those without HUA. Compared to participants with normal SUA, participants with HUA had significantly higher WC; BMI; WHtR; systolic blood pressure (SBP); diastolic blood pressure (DBP); TG, LDL-C, BUN, and Cre levels; AIP; CMI; VAI; LAP index; TyG index; ABSI; and BRI (all $P<0.05$ ). No significant differences were found in FPG, TC, and HDL-C levels between the two groups.

The partial correlation coefficients between the different indices and SUA are shown in Table 2. SUA levels were significantly correlated with the TyG index, AIP, CMI, VAI, and LAP index after adjusting for age in both males and females (all $P<0.05$ ). Among them, the SUA level had the strongest positive correlation with the TyG index in all participants $(\mathrm{r}=$ $0.332,0.229$, and 0.4 , respectively; all $P<0.05$ ), whereas the CMI, VAI, and LAP index had a relatively high correlation. SUA levels were significantly correlated with WC, WHtR, ABSI, and BRI in females only.

Multivariate logistic regression revealed the odds ratios (ORs) and $95 \%$ confidence intervals (CIs) for HUA according to sexspecific TyG index, BMI, WC, WHtR, AIP, CMI, VAI, LAP index, ABSI, and BRI quartiles. After full adjustment for age, SBP, DBP, and selected biochemical indices in model 3, compared with the first quartile, the other three quartiles of visceral obesity TyG index were strongly associated with HUA in both males and females (all $P<0.05$ ). For males and females, the ORs for HUA in the upper quartile of the TyG index were 2.098 (95\% CI, 1.555-2.831) and 7.788 (95\% CI, 3.581-16.937), respectively. The adjusted relative risk of HUA increased with increasing TyG quartiles. The results are presented in Tables 3, 4.

The ROC curves of the different indices for HUA are presented in Table 5 and Figure 1. For males, the TyG index, AIP, CMI, VAI, and LAP index were able to discriminate HUA, and the TyG index showed the highest area under the curve (AUC) value of 0.586 (95\% CI, 0.557-0.614; $P<0.05$ ), with a cutoff value of 8.353 according to the maximum Youden index of 0.133. For females, all indices, except BMI, could discriminate HUA. The CMI showed the highest AUC value of 0.737 (95\% CI, $0.691-0.782 ; P<0.05)$, with a cutoff value of 0.595 according to the maximum Youden index of 0.384. Meanwhile, the TyG index, AIP, VAI, and LAP index had a relatively high AUC value.

\section{DISCUSSION}

\section{Main Findings}

This study investigated and compared the predictive strength of the TyG index and nine obesity indices reflecting general (BMI), abdominal (WC, WHtR), and visceral (AIP, CMI, VAI, LAP index, ABSI, BRI) obesity in the assessment of HUA in a medical checkup population in Xinjiang, China. The main findings of this study were as follows: (1) the TyG index was significantly associated with HUA, and the association remained significant after controlling for age, SBP, DBP, and selected biochemical indices. Further stratification showed that when the TyG index was higher (in the third and fourth quartiles), the risk of developing HUA was higher (2). Some visceral obesity indices were also associated with HUA, but were inferior to the TyG index (3). The TyG index was a better predictor of HUA, especially in females. Therefore, the TyG index may be the best choice for the HUA risk screening index for this regional population. 
TABLE 1 | Clinical characteristics of study participants with and without hyperuricemia.

\begin{tabular}{|c|c|c|c|c|}
\hline Variables & Non-hyperuricemia $(n=1471)$ & Hyperuricemia (n = 772) & $\chi^{2 / t / z}$ & $P$-value \\
\hline Males, n (\%) & $1001(68.05)$ & 615 (79.66) & 33.91 & $<0.001$ \\
\hline Age (years) & $42.44 \pm 12.24$ & $45.17 \pm 12.28$ & -5.014 & $<0.001$ \\
\hline $\mathrm{SBP}(\mathrm{mmHg})$ & $121.36 \pm 17.83$ & $123.14 \pm 17.87$ & -2.252 & 0.024 \\
\hline $\mathrm{DBP}(\mathrm{mmHg})$ & $78.95 \pm 11.59$ & $80.65 \pm 13.33$ & -3.129 & 0.002 \\
\hline SUA (mg/dL) & $4.72 \pm 1.28$ & $8.20 \pm 1.29$ & -60.870 & $<0.001$ \\
\hline $\mathrm{FPG}(\mathrm{mmol} / \mathrm{L})$ & $5.13 \pm 1.38$ & $5.13 \pm 1.80$ & -0.020 & 0.984 \\
\hline $\mathrm{TC}(\mathrm{mmol} / \mathrm{L})$ & $4.50 \pm 1.84$ & $4.44 \pm 1.56$ & 0.749 & 0.454 \\
\hline $\mathrm{TG}(\mathrm{mmol} / \mathrm{L})$ & $1.28(0.86-2.1)$ & $1.85(1.2-3.18)$ & -11.603 & $<0.001$ \\
\hline HDL-C (mmol/L) & $1.19 \pm 0.51$ & $1.17 \pm 0.41$ & 0.491 & 0.623 \\
\hline LDL-C (mmol/L) & $2.67(2.1-3.33)$ & $2.87(2.3-3.49)$ & -4.030 & $<0.001$ \\
\hline BUN (mmol/L) & $5.33 \pm 3.32$ & $5.73 \pm 3.09$ & -2.767 & 0.006 \\
\hline Cre (mL/min) & $80.38 \pm 41.72$ & $86.08 \pm 34.17$ & -3.261 & 0.001 \\
\hline TyG index & $8.61 \pm 0.73$ & $8.96 \pm 0.71$ & -10.802 & $<0.001$ \\
\hline \multicolumn{5}{|c|}{ General obesity index } \\
\hline $\mathrm{BMI}\left(\mathrm{kg} / \mathrm{m}^{2}\right)$ & 25.01 (22.65-27.68) & $25.51(23.18-27.85)$ & -2.739 & 0.017 \\
\hline \multicolumn{5}{|c|}{ Abdominal obesity indices } \\
\hline WC (cm) & $89.48 \pm 12.89$ & $91.44 \pm 13.70$ & -3.339 & 0.001 \\
\hline WHtR & $0.53 \pm 0.08$ & $0.54 \pm 0.09$ & -3.022 & 0.003 \\
\hline \multicolumn{5}{|c|}{ Visceral obesity indices } \\
\hline AIP & $0.09 \pm 0.34$ & $0.24 \pm 0.34$ & -9.887 & $<0.001$ \\
\hline $\mathrm{CMI}$ & $0.6(0.36-1.18)$ & $0.9(0.54-1.72)$ & -9.792 & $<0.001$ \\
\hline VAl & $1.72(1.07-3.1)$ & $2.38(1.44-4.42)$ & -8.387 & $<0.001$ \\
\hline LAP index & 32.19 (18.48-59.8) & 46.2 (27.39-87.48) & -9.146 & $<0.001$ \\
\hline ABSI & $0.80 \pm 0.07$ & $0.81 \pm 0.09$ & -2.977 & 0.003 \\
\hline $\mathrm{BRI}$ & $4.11 \pm 1.57$ & $4.34 \pm 1.92$ & -3.073 & 0.002 \\
\hline
\end{tabular}

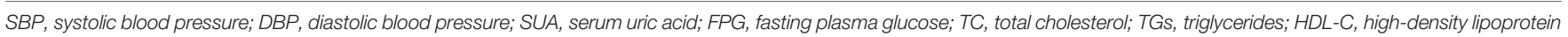
cholesterol; LDL-C, low-density lipoprotein cholesterol; BUN, blood urea nitrogen; Cre, creatinine; TyG index, triglyceride glucose index; BMI, body mass index; WC, waist circumference;

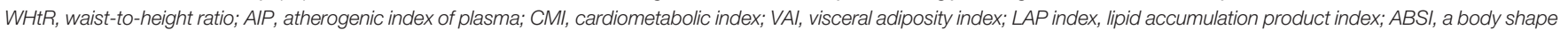
index; BRl, body roundness index.

\section{General and Abdominal Obesity Indices With Hyperuricemia (HUA)}

In many previous studies, obesity was often reflected in BMI, WC, and WHtR, and some studies have shown that these indices are associated with HUA (15). However, BMI reflects only the degree of obesity in humans. Although WC and WHtR can reflect abdominal obesity, they cannot distinguish subcutaneous fat from visceral fat $(16,17)$, and the accumulation of visceral fat is more likely to lead to disorders of uric acid metabolism than subcutaneous fat (18). However, BMI, WC, and WHtR were not significantly associated with HUA in the present study.
The general obesity indices for this population may not be the most appropriate way to assess HUA, which is characterized by excessive weight and fat accumulation. There were differences in body fat distribution between the ethnic groups (19). According to the World Health Organization guidelines, thresholds for BMI, WC, and WHtR are recommended for several different ethnicities and populations (20).

\section{Visceral Obesity Indices With HUA}

Studies have shown that obesity, especially visceral obesity, is closely associated with HUA. However, there is no definitive

TABLE 2 | Partial correlation coefficients between different indices and SUA.

\begin{tabular}{|c|c|c|c|c|c|c|}
\hline \multirow[t]{2}{*}{ Variables } & \multicolumn{2}{|c|}{ Total } & \multicolumn{2}{|c|}{ Males } & \multicolumn{2}{|c|}{ Females } \\
\hline & $r$ & $P$-value & $r$ & $P$-value & $r$ & $P$-value \\
\hline TyG index & 0.332 & $<0.001$ & 0.229 & $<0.001$ & 0.4 & $<0.001$ \\
\hline $\mathrm{BMl}$ & 0.049 & 0.021 & 0.014 & 0.563 & 0.051 & 0.2 \\
\hline WC & 0.118 & $<0.001$ & 0.029 & 0.247 & 0.199 & $<0.001$ \\
\hline WHtR & 0.079 & $<0.001$ & 0.034 & 0.178 & 0.188 & $<0.001$ \\
\hline AIP & 0.31 & $<0.001$ & 0.198 & $<0.001$ & 0.41 & $<0.001$ \\
\hline $\mathrm{CMl}$ & 0.226 & $<0.001$ & 0.151 & $<0.001$ & 0.321 & $<0.001$ \\
\hline VAl & 0.198 & $<0.001$ & 0.152 & $<0.001$ & 0.316 & $<0.001$ \\
\hline LAP index & 0.198 & $<0.001$ & 0.126 & $<0.001$ & 0.331 & $<0.001$ \\
\hline $\mathrm{ABSI}$ & 0.081 & $<0.001$ & 0.014 & 0.573 & 0.243 & $<0.001$ \\
\hline $\mathrm{BRI}$ & 0.055 & 0.009 & 0.018 & 0.467 & 0.161 & $<0.001$ \\
\hline
\end{tabular}

TyG index, triglyceride glucose index; BMI, body mass index; WC, waist circumference; WHtR, waist-to-height ratio; AIP, atherogenic index of plasma; CMI, cardiometabolic index; VAl, visceral adiposity index; LAP index, lipid accumulation product index; ABSI, a body shape index; BRI, body roundness index. 
TABLE 3 | Multivariate logistic regression of different indices for HUA (males).

\begin{tabular}{|c|c|c|c|c|c|c|c|}
\hline \multirow[t]{2}{*}{ Variables } & \multirow[t]{2}{*}{ Quartile 1} & \multicolumn{2}{|c|}{ Quartile 2} & \multicolumn{2}{|c|}{ Quartile 3} & \multicolumn{2}{|c|}{ Quartile 4} \\
\hline & & OR (95\% Cl) & $P$-value & OR (95\% Cl) & $P$-value & OR (95\% Cl) & $P$-value \\
\hline TyG index & $\leq 8.33$ & 8.34-8.78 & & 8.79-9.34 & & $\geq 9.35$ & \\
\hline Model 1 & Reference & $\begin{array}{c}1.573(1.169- \\
2.116)\end{array}$ & 0.003 & $\begin{array}{c}1.818(1.355- \\
2.441)\end{array}$ & $<0.001$ & $\begin{array}{c}2.202(1.643- \\
2.952)\end{array}$ & $<0.001$ \\
\hline Model 2 & Reference & $\begin{array}{c}1.519(1.127- \\
2.047)\end{array}$ & 0.006 & $1.748(1.3-2.351)$ & $<0.001$ & $\begin{array}{c}2.131(1.585- \\
2.867)\end{array}$ & $<0.001$ \\
\hline Model 3 & Reference & $\begin{array}{c}1.501(1.108- \\
2.032)\end{array}$ & 0.009 & $\begin{array}{c}1.762(1.303- \\
2.383)\end{array}$ & $<0.001$ & $\begin{array}{c}2.098(1.555- \\
2.831)\end{array}$ & $<0.001$ \\
\hline $\mathrm{BMl}$ & $\leq 23$ & 24-25 & & 26-28 & & $\geq 29$ & \\
\hline Model 1 & Reference & $1.277(0.959-1.7)$ & 0.094 & $\begin{array}{c}1.452(1.089- \\
1.935)\end{array}$ & 0.011 & $1.15(0.861-1.535)$ & 0.343 \\
\hline Model 2 & Reference & $\begin{array}{c}1.221(0.911- \\
1.635)\end{array}$ & 0.182 & $1.351(1.003-1.82)$ & 0.048 & $1.029(0.757-1.4)$ & 0.855 \\
\hline Model 3 & Reference & $\begin{array}{c}1.211(0.901- \\
1.629)\end{array}$ & 0.205 & 1.369 (1.013-1.85) & 0.041 & $\begin{array}{c}1.045(0.767- \\
1.424)\end{array}$ & 0.781 \\
\hline WC & $\leq 82$ & 83-90 & & $91-99$ & & $\geq 100$ & \\
\hline Model 1 & Reference & $1.164(0.88-1.539)$ & 0.287 & $\begin{array}{c}1.189(0.893- \\
1.584)\end{array}$ & 0.236 & $1.04(0.78-1.387)$ & 0.79 \\
\hline Model 2 & Reference & $\begin{array}{c}1.116(0.839- \\
1.483)\end{array}$ & 0.451 & $1.077(0.8-1.45)$ & 0.626 & $\begin{array}{c}0.914(0.673- \\
1.241)\end{array}$ & 0.564 \\
\hline Model 3 & Reference & $\begin{array}{c}1.128(0.846- \\
1.506)\end{array}$ & 0.412 & $\begin{array}{c}1.091(0.807- \\
1.474)\end{array}$ & 0.573 & $\begin{array}{c}0.941(0.691- \\
1.281)\end{array}$ & 0.697 \\
\hline WHtR & $\leq 0.49$ & $0.5-0.53$ & & $0.54-0.59$ & & $\geq 0.6$ & \\
\hline Model 1 & Reference & $1.1(0.827-1.463)$ & 0.513 & $1.195(0.9-1.588)$ & 0.219 & $\begin{array}{c}1.055(0.792- \\
1.404)\end{array}$ & 0.715 \\
\hline Model 2 & Reference & $\begin{array}{c}1.042(0.779- \\
1.394)\end{array}$ & 0.78 & $\begin{array}{c}1.093(0.814- \\
1.468)\end{array}$ & 0.555 & $\begin{array}{c}0.927(0.683- \\
1.258)\end{array}$ & 0.627 \\
\hline Model 3 & Reference & $1.03(0.767-1.382)$ & 0.845 & $\begin{array}{c}1.109(0.823- \\
1.495)\end{array}$ & 0.496 & $\begin{array}{c}0.945(0.695- \\
1.285)\end{array}$ & 0.717 \\
\hline AIP & $\leq-0.04$ & $-0.03-0.18$ & & $0.19-0.45$ & & $\geq 0.46$ & \\
\hline Model 1 & Reference & $\begin{array}{c}1.475(1.102- \\
1.974)\end{array}$ & 0.009 & $\begin{array}{c}1.531(1.145- \\
2.048)\end{array}$ & 0.004 & $\begin{array}{c}1.869(1.399- \\
2.497)\end{array}$ & $<0.001$ \\
\hline Model 2 & Reference & $\begin{array}{c}1.444(1.077- \\
1.938)\end{array}$ & 0.014 & $1.5(1.119-2.011)$ & 0.007 & $\begin{array}{c}1.819(1.355- \\
2.444)\end{array}$ & $<0.001$ \\
\hline Model 3 & Reference & $\begin{array}{c}1.418(1.054- \\
1.908)\end{array}$ & 0.021 & $1.502(1.116-2.02)$ & 0.007 & 1.839 (1.364-2.48) & $<0.001$ \\
\hline CMl & $\leq 0.47$ & $0.48-0.8$ & & $0.81-1.54$ & & $\geq 1.55$ & \\
\hline Model 1 & Reference & $\begin{array}{c}1.454(1.083- \\
1.953)\end{array}$ & 0.013 & $\begin{array}{c}1.896(1.417- \\
2.536)\end{array}$ & $<0.001$ & $1.808(1.35-2.421)$ & $<0.001$ \\
\hline Model 2 & Reference & $\begin{array}{c}1.407(1.045- \\
1.895)\end{array}$ & 0.024 & 1.85 (1.379-2.483) & $<0.001$ & $\begin{array}{c}1.739(1.289- \\
2.346)\end{array}$ & $<0.001$ \\
\hline Model 3 & Reference & $1.39(1.029-1.877)$ & 0.032 & $\begin{array}{c}1.854(1.376- \\
2.499)\end{array}$ & $<0.001$ & $\begin{array}{c}1.752(1.292- \\
2.374)\end{array}$ & $<0.001$ \\
\hline VAl & $\leq 1.19$ & $1.2-1.98$ & & $1.99-3.74$ & & $\geq 3.75$ & \\
\hline Model 1 & Reference & $\begin{array}{c}1.383(1.031- \\
1.855)\end{array}$ & 0.031 & $\begin{array}{c}1.883(1.409- \\
2.516)\end{array}$ & $<0.001$ & $\begin{array}{c}1.668(1.246- \\
2.231)\end{array}$ & 0.001 \\
\hline Model 2 & Reference & $\begin{array}{c}1.349(1.003- \\
1.814)\end{array}$ & 0.048 & $1.82(1.358-2.44)$ & $<0.001$ & $\begin{array}{c}1.608(1.194- \\
2.165)\end{array}$ & 0.002 \\
\hline Model 3 & Reference & $1.33(0.986-1.794)$ & 0.062 & $\begin{array}{c}1.822(1.354- \\
2.453)\end{array}$ & $<0.001$ & $\begin{array}{c}1.615(1.194- \\
2.186)\end{array}$ & 0.002 \\
\hline LAP index & $\leq 21.6$ & 21.61-39.96 & & $39.97-77.14$ & & $\geq 77.15$ & \\
\hline Model 1 & Reference & $\begin{array}{c}1.821(1.356- \\
2.445)\end{array}$ & $<0.001$ & $\begin{array}{c}1.758(1.309- \\
2.362)\end{array}$ & $<0.001$ & $2.091(1.56-2.803)$ & $<0.001$ \\
\hline Model 2 & Reference & $1.761(1.303-2.38)$ & $<0.001$ & $\begin{array}{c}1.709(1.261- \\
2.318)\end{array}$ & 0.001 & $2.016(1.48-2.747)$ & $<0.001$ \\
\hline Model 3 & Reference & $\begin{array}{c}1.787(1.317- \\
2.425)\end{array}$ & $<0.001$ & $\begin{array}{c}1.749(1.283- \\
2.383)\end{array}$ & $<0.001$ & $\begin{array}{c}2.043(1.493- \\
2.794)\end{array}$ & $<0.001$ \\
\hline ABSI & $\leq 0.76$ & $0.77-0.8$ & & $0.81-0.84$ & & $\geq 0.85$ & \\
\hline Model 1 & Reference & $\begin{array}{c}0.895(0.669- \\
1.197)\end{array}$ & 0.454 & $\begin{array}{c}1.351(1.021- \\
1.788)\end{array}$ & 0.035 & $\begin{array}{c}1.351(1.012- \\
1.788)\end{array}$ & 0.827 \\
\hline Model 2 & Reference & $\begin{array}{c}0.872(0.651- \\
1.168)\end{array}$ & 0.359 & $\begin{array}{c}1.291(0.973- \\
1.713)\end{array}$ & 0.077 & $\begin{array}{c}0.968(0.722- \\
1.296)\end{array}$ & 0.825 \\
\hline
\end{tabular}


TABLE 3 | Continued

\begin{tabular}{|c|c|c|c|c|c|c|c|}
\hline \multirow[t]{2}{*}{ Variables } & \multirow[t]{2}{*}{ Quartile 1} & \multicolumn{2}{|c|}{ Quartile 2} & \multicolumn{2}{|c|}{ Quartile 3} & \multicolumn{2}{|c|}{ Quartile 4} \\
\hline & & OR $(95 \% \mathrm{Cl})$ & $P$-value & OR (95\% Cl) & $P$-value & OR (95\% Cl) & $P$-value \\
\hline Model 3 & Reference & $\begin{array}{c}0.867(0.645- \\
1.166)\end{array}$ & 0.346 & $1.3(0.976-1.73)$ & 0.073 & $\begin{array}{c}0.981(0.729- \\
1.319)\end{array}$ & 0.899 \\
\hline $\mathrm{BRI}$ & $\leq 3.08$ & 3.09-3.93 & & $3.94-5.11$ & & $\geq 5.12$ & \\
\hline Model 1 & Reference & $1.1(0.827-1.463)$ & 0.513 & $1.195(0.9-1.588)$ & 0.219 & $\begin{array}{c}1.055(0.792- \\
1.404)\end{array}$ & 0.715 \\
\hline Model 2 & Reference & $\begin{array}{c}1.042(0.779- \\
1.394)\end{array}$ & 0.78 & $\begin{array}{c}1.093(0.814- \\
1.468)\end{array}$ & 0.555 & $\begin{array}{c}0.927(0.683- \\
1.258)\end{array}$ & 0.627 \\
\hline Model 3 & Reference & $1.03(0.767-1.382)$ & 0.845 & $\begin{array}{c}1.109(0.823- \\
1.495)\end{array}$ & 0.496 & $\begin{array}{c}0.945(0.695- \\
1.285)\end{array}$ & 0.717 \\
\hline
\end{tabular}

Model 1: unadjusted; model 2: adjusted for age, SBP, and DBP; model 3: adjusted for all variables in model 2 plus BUN, Cre, TC, and LDL-C. TyG index, triglyceride glucose index; BMI, body mass index; WC, waist circumference; WHtR, waist-to-height ratio; AIP, atherogenic index of plasma; CMI, cardiometabolic index; VAl, visceral adiposity index; LAP index, lipid accumulation product index; $A B S I$, a body shape index; $B R I$, body roundness index.

conclusion regarding which criterion is more valuable in reflecting visceral fat content and predicting HUA. One of the important reasons for this is that there are some limitations to the various criteria for measuring obesity. New indices, such as the ABSI and BRI, have been presented to distribute adipose tissue more precisely, and some studies have reported a close association between these indices and $\operatorname{HUA}(21,22)$. In contrast to previous studies, no correlation between these indices and HUA was found in the present study. The reasons for this different conclusion may include the following: (1) participants from different areas had different lifestyles and variations in age distribution and sex composition. (2) The ABSI and BRI cannot distinguish between the distribution of subcutaneous and visceral adipose tissues (23). (3) The ABSI was originally established to predict mortality in a follow-up cohort (24), whereas this study utilized it in a cross-sectional manner. This may be the reason for the low screening value.

Previous studies have shown that indices consisting of TG were well suited to identify individuals with unhealthy metabolism (25). The CMI is a new index for evaluating visceral obesity using lipid parameters TG and HDL-C to waist height ratio, which has been shown to be associated with several metabolic syndromes $(26,27)$. The VAI integrates the traditional obesity indices WC and BMI with TG and HDL-C, which can better indicate visceral fat content and its distribution (28). The LAP index integrates WC, an indicator of abdominal obesity, and TG, which is closely related to visceral fat distribution (6). The present study showed that the CMI, VAI, and LAP index could affect uric acid levels and increase the risk of HUA, which is consistent with the results of previous studies (29). Compared with traditional anthropometric indices, the CMI, VAI, and LAP index have better predictive power for HUA in both males and females. In particular, the sensitivity of the ROC curve was higher in females than in males. This may be related to the fact that the CMI, LAP index, and VAI fully integrate WC, an indicator for assessing abdominal obesity, and TG, which is related to the visceral fat distribution. This suggests that higher visceral fat accumulation expressed by elevated LAP has a greater effect on uric acid metabolism than BMI values, which includes less specificity for subcutaneous fat accumulation. Visceral fat promotes the synthesis of phosphoribosyl pyrophosphate from very low-density lipoprotein and ribose 5-phosphate, which leads to the excessive production of uric acid. Meanwhile, the VAI was a better predictor of HUA than BMI, WC, and WHR independently in the Chinese population (30), probably because of the accumulation of visceral fat, which allows free fatty acids (FFAs) to enter the liver via the portal vein, increasing the synthesis of TGs and causing hypertriglyceridemia. Moreover, FFAs can increase the synthesis of purines via the pentose phosphate pathway. The correlation between the CMI, VAI, and LAP index and uric acid in this study further confirmed the association between visceral fat and HUA.

The AIP is a sensitive index of lipid metabolism disorders proposed by Dobiasova et al. (31), which uses log (TG/HDL-C) as an index that can be used as a predictor of the risk of plasma atherosclerosis development, and its value is negatively correlated with LDL particle size and LDL-C as a routine index of clinical testing if abnormally elevated, leading to a high risk of cardiovascular disease. The AIP has been significantly associated with HUA, hypercholesterolemia, hyperlipidemia, and metabolic syndrome, which are all risk factors for cardiovascular disease (32). Zhu et al. found a positive association between higher levels of AIP, a new biomarker associated with obesity (33). In the present study, the partial correlation analysis suggested that the AIP was positively associated with SUA and could be used as an independent risk factor for predicting HUA in both sexes. This is consistent with the results of a cross-sectional study by Chang et al. on the association between blood uric acid and AIP in a population from northeastern China (34). The mechanism may be due to the fact that changes in cholesterol and TG can cause disorders of lipid metabolism, whereas the association between dyslipidemia and HUA is bidirectional. On the one hand, due to the long and cold winter in Xinjiang, the local population has a high proportion of high-purine and high-fat diet intake, which easily causes fat accumulation and disorders of lipid and purine metabolism. On the other hand, high concentrations of uric acid can affect lipid peroxidation and LDL cholesterol oxidation, reducing the activity of the corresponding enzymes and decreasing cholesterol catabolism, causing changes in blood lipids (35). 
TABLE 4 | Multivariate logistic regression of different indices for HUA (females).

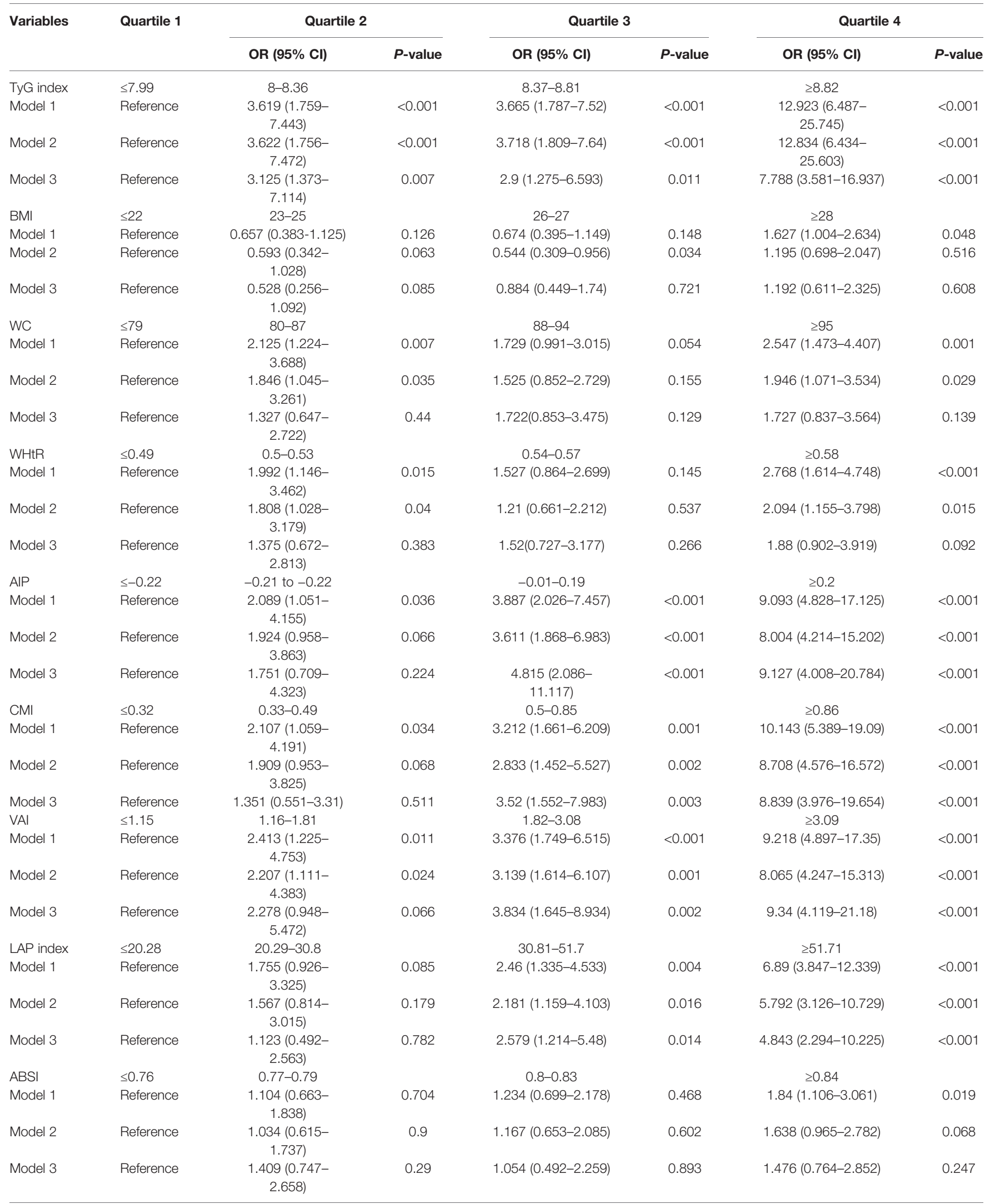


TABLE 4 | Continued

\begin{tabular}{|c|c|c|c|c|c|c|c|}
\hline \multirow[t]{2}{*}{ Variables } & \multirow[t]{2}{*}{ Quartile 1} & \multicolumn{2}{|c|}{ Quartile 2} & \multicolumn{2}{|c|}{ Quartile 3} & \multicolumn{2}{|c|}{ Quartile 4} \\
\hline & & OR $(95 \% \mathrm{Cl})$ & $P$-value & OR $(95 \% \mathrm{Cl})$ & $P$-value & OR $(95 \% \mathrm{Cl})$ & $P$-value \\
\hline $\mathrm{BRI}$ & $\leq 3.12$ & $3.13-4.03$ & & $4.04-4.82$ & & $\geq 4.83$ & \\
\hline Model 1 & Reference & $\begin{array}{c}1.992(1.146- \\
3.462)\end{array}$ & 0.015 & 1.527 (0.864-2.699) & 0.145 & $2.768(1.614-4.748)$ & $<0.001$ \\
\hline Model 2 & Reference & $\begin{array}{c}1.808(1.028- \\
3.179)\end{array}$ & 0.04 & $1.21(0.661-2.212)$ & 0.537 & $2.094(1.155-3.798)$ & 0.015 \\
\hline Model 3 & Reference & $\begin{array}{c}1.375(0.672- \\
2.813)\end{array}$ & 0.383 & $1.52(0.727-3.177)$ & 0.266 & 1.88 (0.902-3.919) & 0.092 \\
\hline
\end{tabular}

Model 1: unadjusted; model 2: adjusted for age, SBP, and DBP; model 3: adjusted for all variables in model 2 plus BUN, Cre, TC, and LDL-C. TyG index, triglyceride glucose index; BMI,

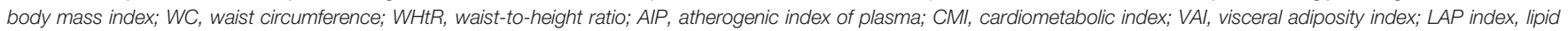
accumulation product index; $A B S I$, a body shape index; $B R I$, body roundness index.

\section{The Triglyceride Glucose Index With HUA}

Epidemiological studies have established a significant association between IR and SUA $(36,37)$, and compensatory hyperinsulinemia that occurs after IR can reduce uric acid excretion by renal tubular sodium reabsorption and cause HUA (38). Conversely, higher uric acid levels can reduce nitric oxide bioavailability and mitochondrial oxidative stress, leading to IR (39). Therefore, the evaluation of IR status in a normal physical examination population is beneficial not only for uric acid control but also for the prevention of other metabolic diseases. Traditional IR assessment tools, such as the high insulin glucose clamp test and steady-state mode assessment method, cannot be commonly used because of their complicated, invasive, and expensive characteristics. In recent years, several simple IR assessment tools have been developed and used clinically. The TyG index was developed in 2008 by Simental-Mendía et al. (40) and has better sensitivity and specificity compared to the gold standard for
IR detection (41). Some studies have reported that the TyG index is associated with atherosclerosis, metabolic syndrome, and type 2 diabetes mellitus (42-44). Mazidi et al. (9) found a correlation between the TyG index and HUA in Caucasian populations. In the present study, logistic regression models revealed a significant correlation between the TyG index and the risk of HUA in both males and females compared to other obesity indices, even after multivariate adjustment. Moreover, this correlation was consistent across all subgroups after performing a stratified analysis of the TyG index, and the risk of HUA was higher in the high quartile than in the low quartile. The TyG index also had a significant value in identifying HUA in both sexes in the ROC analysis compared to other indices $(P<0.001)$, especially in females. This may be due to the fact that estrogen, as a uric acid producing agent, leads to more complex endocrine factors in its metabolism than in males, as well as differences in lipid metabolism between the sexes. This finding is consistent with the results of a cross-sectional study by Shi et al.

TABLE 5 | Comparison of the ability of different indices to predict HUA.

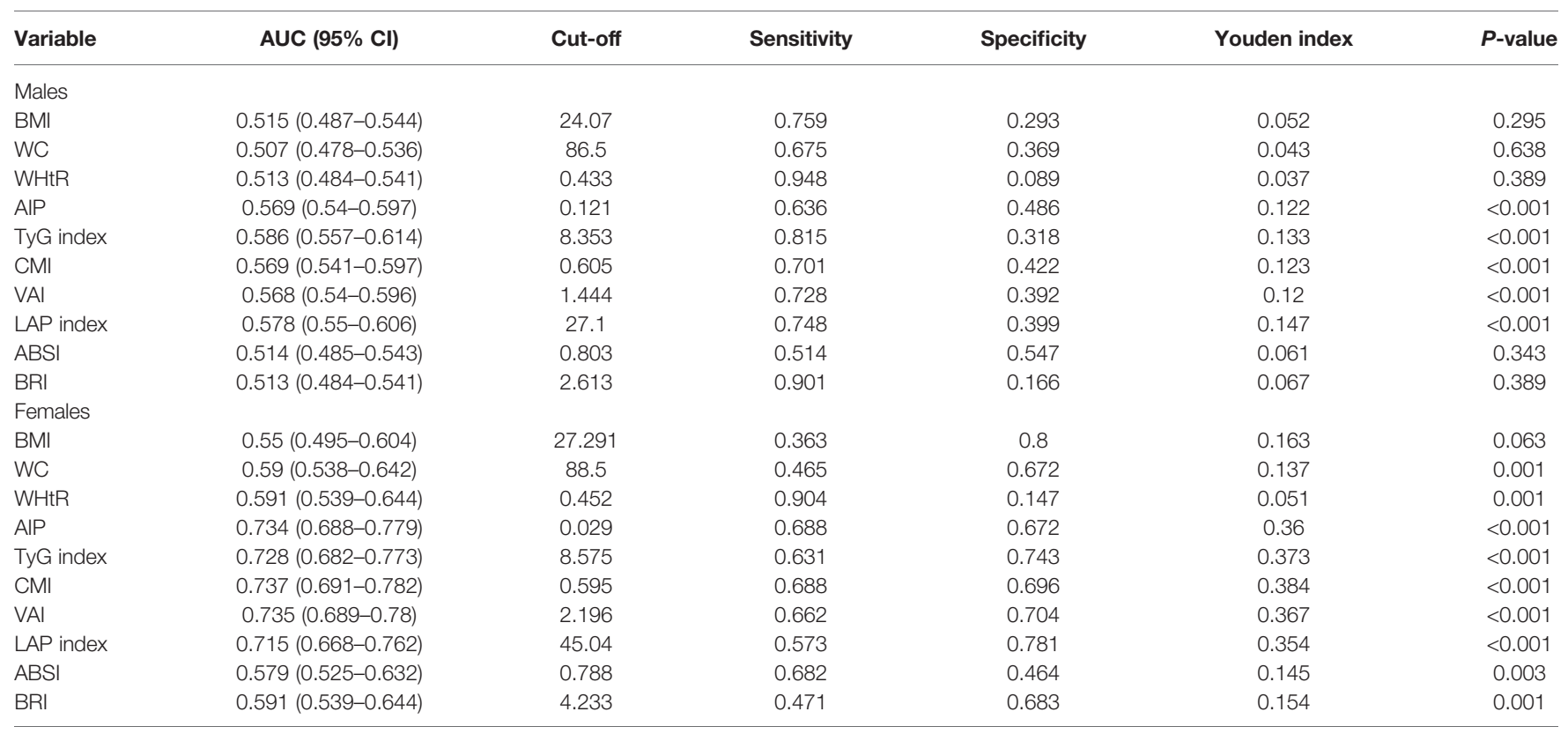

TyG index, triglyceride glucose index; BMI, body mass index; WC, waist circumference; WHtR, waist-to-height ratio; AIP, atherogenic index of plasma; CMI, cardiometabolic index; VAI, visceral adiposity index; LAP index, lipid accumulation product index; ABSI, a body shape index; BRI, body roundness index. 

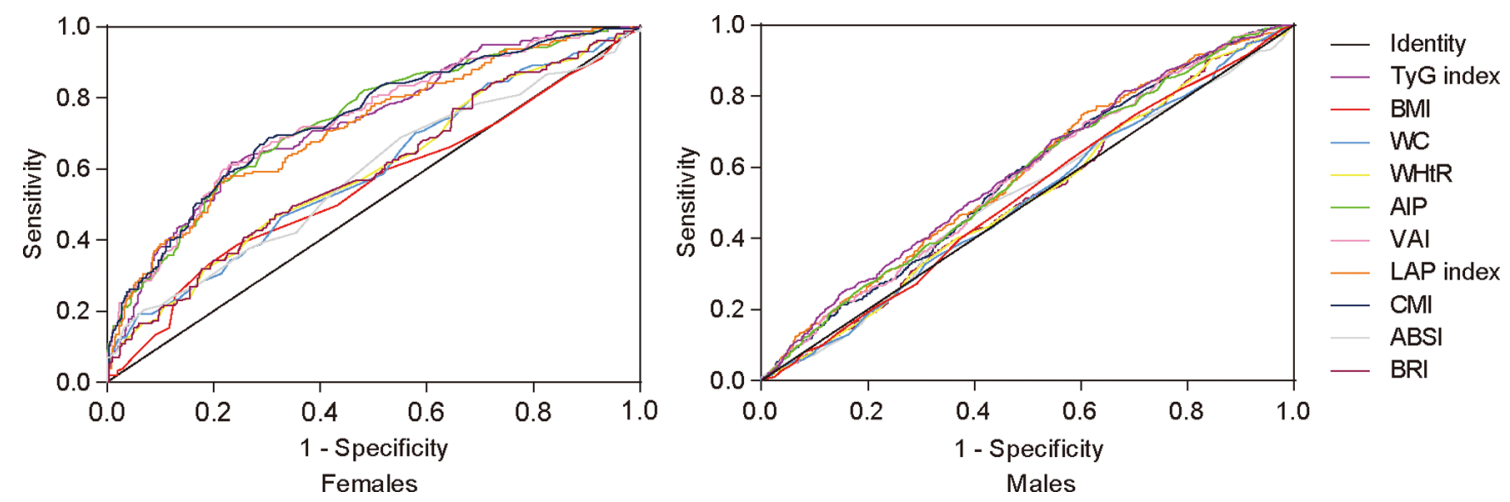

FIGURE 1 | Receiver operating characteristic curve analysis by sex. TyG index, triglyceride glucose index; BMI, body mass index; WC, waist circumference; WHtR, waist-to-height ratio; AIP, atherogenic index of plasma; CMI, cardiometabolic index; VAI, visceral adiposity index; LAP index, lipid accumulation product index; ABSI, a body shape index; BRI, body roundness index.

(45) in northeastern China. One possible mechanism is that IR affects lipid metabolism by decreasing lipoprotein lipase activity and reducing lipocalin production (4). Second, high TG levels are degraded to FFAs, which are transported to other tissues and accelerate adenosine triphosphate breakdown. Abnormal lipid metabolism also impairs the kidney, decreases renal blood flow, reduces urinary uric acid excretion, and increases SUA levels (46).

This study systematically investigated the predictive value of the TyG index and nine obesity indices for HUA and included a comprehensive range of indices. All data in the study were obtained from the same regional population, which reduced the risk of bias in the sample source. However, this study has some limitations. First, it investigated a specific region, Barkol County, which may not best represent the overall status of ethnicity and related diseases in Xinjiang. Second, it has been previously reported that uric acid levels can be affected by diet, but information on dietary patterns, such as dairy and meat intake, was not analyzed in this study population, this may lead to biased results. Third, the cross-sectional survey could not clearly determine the causal association between the risk factors and the incidence of HUA. Systematic large-scale studies are required to further elucidate the changes in uric acid levels and their associated risk factors.

\section{CONCLUSIONS}

In conclusion, different indices were differentially related to HUA and had different predictive abilities. In addition to generalized obesity, abdominal obesity and the resulting visceral fat accumulation also need to be considered. The TyG index was more closely related to HUA than the obesity indices in the medical checkup population in Xinjiang, China. Thus, it can be used as an important index for HUA risk screening and population health management.

\section{DATA AVAILABILITY STATEMENT}

The original contributions presented in the study are included in the article/supplementary material. Further inquiries can be directed to the corresponding authors.

\section{ETHICS STATEMENT}

The studies involving human participants were reviewed and approved by The Medical Ethics Committee of the First Affiliated Hospital of Xinjiang Medical University. The patients/ participants provided their written informed consent to participate in this study.

\section{AUTHOR CONTRIBUTIONS}

YS and $\mathrm{CH}$ contributed to conception and design of the study. $\mathrm{BZ}, \mathrm{WC}, \mathrm{MC}$, and TT organized the database. ML, YH, RL performed the statistical analysis. MK wrote the first draft of the manuscript. All authors contributed to manuscript revision, read, and approved the submitted version.

\section{FUNDING}

This study was funded by the National Natural Science Foundation of China (No.81960169,81760169, and 81900795), the Natural Science Foundation of the Xinjiang Uygur Autonomous Region (No. 2019D01C219 and 2021D01C275), the Project of Scientific Research Program for Universities in Xinjiang Uygur Autonomous Region(No.XJEDU2021Y054), the Postgraduate Research Innovation Project in Xinjiang Uygur Autonomous Region(XJ2021G228). 


\section{REFERENCES}

1. Liu R, Han C, Wu D, Xia X, Gu J, Guan H, et al. Prevalence of Hyperuricemia and Gout in Mainland China From 2000 to 2014: A Systematic Review and Meta-Analysis. BioMed Res Int (2015) 2015:762820. doi: 10.1155/2015/ 762820

2. Zhu Y, Pandya BJ, Choi HK. Prevalence of Gout and Hyperuricemia in the Us General Population: The National Health and Nutrition Examination Survey 2007-2008. Arthritis Rheum (2011) 63(10):3136-41. doi: 10.1002/art.30520

3. Kim Y, Kang J, Kim GT. Prevalence of Hyperuricemia and Its Associated Factors in the General Korean Population: An Analysis of a Population-Based Nationally Representative Sample. Clin Rheumatol (2018) 37(9):2529-38. doi: 10.1007/s10067-018-4130-2

4. Wang H, Zhang H, Sun L, Guo W. Roles of Hyperuricemia in Metabolic Syndrome and Cardiac-Kidney-Vascular System Diseases. Am J Transl Res (2018) 10(9):2749-63.

5. Katsiki N, Dimitriadis GD, Mikhailidis DP. Serum Uric Acid and Diabetes: From Pathophysiology to Cardiovascular Disease. Curr Pharm Des (2021) 27 (16):1941-51. doi: 10.2174/1381612827666210104124320

6. Gu D, Ding Y, Zhao Y, Miao S, Qu Q. Positively Increased Visceral Adiposity Index in Hyperuricemia Free of Metabolic Syndrome. Lipids Health Dis (2018) 17(1):101. doi: 10.1186/s12944-018-0761-1

7. Zhu B, Wang J, Chen K, Yan W, Wang A, Wang W, et al. A High Triglyceride Glucose Index Is More Closely Associated With Hypertension Than Lipid or Glycemic Parameters in Elderly Individuals: A Cross-Sectional Survey From the Reaction Study. Cardiovasc Diabetol (2020) 19(1):112. doi: 10.1186/ s12933-020-01077-6

8. Hernández-Díazcouder A, Romero-Nava R, Carbó R, Sánchez-Lozada LG, Sánchez-Muñoz F. High Fructose Intake and Adipogenesis. Int J Mol Sci (2019) 20(11);2787. doi: 10.3390/ijms20112787

9. Mazidi M, Katsiki N, Mikhailidis DP, Banach M. The Link Between Insulin Resistance Parameters and Serum Uric Acid Is Mediated by Adiposity. Atherosclerosis (2018) 270:180-6. doi: 10.1016/j.atherosclerosis.2017.12.033

10. Motamed N, Khonsari MR, Rabiee B, Ajdarkosh H, Hemasi GR, Sohrabi MR, et al. Discriminatory Ability of Visceral Adiposity Index (Vai) in Diagnosis of Metabolic Syndrome: A Population Based Study. Exp Clin Endocrinol Diabetes (2017) 125(3):202-7. doi: 10.1055/s-0042-119032

11. Wang S, Shi J, Peng Y, Fang Q, Mu Q, Gu W, et al. Stronger Association of Triglyceride Glucose Index Than the Homa-Ir With Arterial Stiffness in Patients With Type 2 Diabetes: A Real-World Single-Centre Study. Cardiovasc Diabetol (2021) 20(1):82. doi: 10.1186/s12933-021-01274-X

12. Huang YF, Yang KH, Chen SH, Xie Y, Huang CB, Qing YF, et al. Practice Guideline for Patients With Hyperuricemia/Gout. Zhonghua Nei Ke Za Zhi (2020) 59(7):519-27. doi: 10.3760/cma.j.cn112138-20200505-00449

13. Sheng G, Lu S, Xie Q, Peng N, Kuang M, Zou Y. The Usefulness of Obesity and Lipid-Related Indices to Predict the Presence of Non-Alcoholic Fatty Liver Disease. Lipids Health Dis (2021) 20(1):134. doi: 10.1186/s12944-02101561-2

14. Wakabayashi I. Associations of Smoking and Drinking With New LipidRelated Indices in Women With Hyperglycemia. Womens Health Rep (New Rochelle) (2021) 2(1):23-31. doi: 10.1089/whr.2020.0100

15. Mukhopadhyay P, Ghosh S, Pandit K, Chatterjee P, Majhi B, Chowdhury S. Uric Acid and Its Correlation With Various Metabolic Parameters: A Population-Based Study. Indian J Endocrinol Metab (2019) 23(1):134-9. doi: 10.4103/ijem.IJEM_18_19

16. Haslam DW, James WPT. Obesity. Lancet (2005) 366(9492):1197-209. doi: 10.1016/S0140-6736(05)67483-1

17. Pouliot MC, Després JP, Lemieux S, Moorjani S, Bouchard C, Tremblay A, et al. Waist Circumference and Abdominal Sagittal Diameter: Best Simple Anthropometric Indexes of Abdominal Visceral Adipose Tissue Accumulation and Related Cardiovascular Risk in Men and Women. Am J Cardiol (1994) 73(7):460-8. doi: 10.1016/0002-9149(94)90676-9

18. Yamada A, Sato KK, Kinuhata S, Uehara S, Endo G, Hikita Y, et al. Association of Visceral Fat and Liver Fat With Hyperuricemia. Arthritis Care Res (Hoboken) (2016) 68(4):553-61. doi: 10.1002/acr.22729

19. Barreira TV, Broyles ST, Gupta AK, Katzmarzyk PT. Relationship of Anthropometric Indices to Abdominal and Total Body Fat in Youth: Sex and Race Differences. Obes (Silver Spring) (2014) 22(5):1345-50. doi: 10.1002/ oby. 20714

20. Semlitsch T, Stigler FL, Jeitler K, Horvath K, Siebenhofer A. Management of Overweight and Obesity in Primary Care-A Systematic Overview of International Evidence-Based Guidelines. Obes Rev (2019) 20(9):1218-30. doi: $10.1111 /$ obr.12889

21. Tu CM, Wei TE, Tseng GS, Chen CC, Liu CW. Serum Uric Acid Is Associated With Incident Metabolic Syndrome Independent of Body Shape Index and Body Roundness Index in Healthy Individuals. Nutr Metab Cardiovasc Dis (2021) 31(11):3142-51. doi: 10.1016/j.numecd.2021.07.008

22. Zhang N, Chang Y, Guo X, Chen Y, Ye N, Sun Y. A Body Shape Index and Body Roundness Index: Two New Body Indices for Detecting Association Between Obesity and Hyperuricemia in Rural Area of China. Eur J Intern Med (2016) 29:32-6. doi: 10.1016/j.ejim.2016.01.019

23. Chaudry O, Grimm A, Friedberger A, Kemmler W, Uder M, Jakob F, et al Magnetic Resonance Imaging and Bioelectrical Impedance Analysis to Assess Visceral and Abdominal Adipose Tissue. Obes (Silver Spring) (2020) 28 (2):277-83. doi: 10.1002/oby.22712

24. Krakauer NY, Krakauer JC. A New Body Shape Index Predicts Mortality Hazard Independently of Body Mass Index. PloS One (2012) 7(7):e39504. doi: 10.1371/journal.pone.0039504

25. Yu X, Wang L, Zhang W, Ming J, Jia A, Xu S, et al. Fasting Triglycerides and Glucose Index Is More Suitable for the Identification of Metabolically Unhealthy Individuals in the Chinese Adult Population: A Nationwide Study. J Diabetes Investig (2019) 10(4):1050-8. doi: 10.1111/jdi.12975

26. Wakabayashi I, Daimon T. The "Cardiometabolic Index" as a New Marker Determined by Adiposity and Blood Lipids for Discrimination of Diabetes Mellitus. Clin Chim Acta (2015) 438:274-8. doi: 10.1016/j.cca.2014.08.042

27. Shi WR, Wang HY, Chen S, Guo XF, Li Z, Sun YX. Estimate of Prevalent Diabetes From Cardiometabolic Index in General Chinese Population: A Community-Based Study. Lipids Health Dis (2018) 17(1):236. doi: 10.1186/ s12944-018-0886-2

28. Amato MC, Giordano C, Galia M, Criscimanna A, Vitabile S, Midiri M, et al. Visceral Adiposity Index: A Reliable Indicator of Visceral Fat Function Associated With Cardiometabolic Risk. Diabetes Care (2010) 33(4):920-2. doi: $10.2337 / \mathrm{dc} 09-1825$

29. Zuo YQ, Gao ZH, Yin YL, Yang X, Feng PY. Association Between the Cardiometabolic Index and Hyperuricemia in an Asymptomatic Population With Normal Body Mass Index. Int J Gen Med (2021) 14:8603-10. doi: 10.2147/IJGM.S340595

30. Dong $\mathrm{H}, \mathrm{Xu} \mathrm{Y}$, Zhang X, Tian S. Visceral Adiposity Index Is Strongly Associated With Hyperuricemia Independently of Metabolic Health and Obesity Phenotypes. Sci Rep Sci Rep (2017) 7(1):8822. doi: 10.1038/s41598017-09455-z

31. Dobiásová M, Raslová K, Rauchová H, Vohnout B, Ptácková K, Frohlich J. Atherogenic Lipoprotein Profile in Families With and Without History of Early Myocardial Infarction. Physiol Res (2001) 50(1):1-8.

32. Kammar-García A, López-Moreno P, Hernández-Hernández ME, Ortíz-Bueno AM, Martínez-Montaño MLC. Atherogenic Index of Plasma as a Marker of Cardiovascular Risk Factors in Mexicans Aged 18 to 22 Years. Proc (Bayl Univ Med Cent) (2020) 34(1):22-7. doi: 10.1080/08998280.2020.1799479

33. Zhu X, Yu L, Zhou H, Ma Q, Zhou X, Lei T, et al. Atherogenic Index of Plasma Is a Novel and Better Biomarker Associated With Obesity: A PopulationBased Cross-Sectional Study in China. Lipids Health Dis (2018) 17(1):37. doi: 10.1186/s12944-018-0686-8

34. Chang Y, Li Y, Guo X, Guo L, Sun Y. Atherogenic Index of Plasma Predicts Hyperuricemia in Rural Population: A Cross-Sectional Study From Northeast China. Int J Environ Res Public Health (2016) 13(9):879. doi: 10.3390/ ijerph13090879

35. Ahmadnezhad M, Arefhosseini SR, Parizadeh MR, Tavallaie S, Tayefi M, Darroudi S, et al. Association Between Serum Uric Acid, High Sensitive CReactive Protein and Pro-Oxidant-Antioxidant Balance in Patients With Metabolic Syndrome. BioFactors (2018) 44(3):263-71. doi: 10.1002/biof.1424

36. Adachi SI, Yoshizawa F, Yagasaki K. Hyperuricemia in Type 2 Diabetic Model Kk-A(Y)/Ta Mice: A Potent Animal Model With Positive Correlation Between Insulin Resistance and Plasma High Uric Acid Levels. BMC Res Notes (2017) 10(1):577. doi: 10.1186/s13104-017-2897-x 
37. Zong J, Sun Y, Zhang Y, Yuan J, Wang X, Zhang R, et al. Correlation Between Serum Uric Acid Level and Central Body Fat Distribution in Patients With Type 2 Diabetes. Diabetes Metab Syndr Obes (2020) 13:2521-31. doi: 10.2147/ DMSO.S260891

38. Facchini F, Chen YD, Hollenbeck CB, Reaven GM. Relationship Between Resistance to Insulin-Mediated Glucose Uptake, Urinary Uric Acid Clearance, and Plasma Uric Acid Concentration. JAMA (1991) 266(21):3008-11. doi: 10.1001/jama.1991.03470210076036

39. Lanaspa MA, Sanchez-Lozada LG, Choi YJ, Cicerchi C, Kanbay M, Roncal-Jimenez CA, et al. Uric Acid Induces Hepatic Steatosis by Generation of Mitochondrial Oxidative Stress: Potential Role in Fructose-Dependent and -Independent Fatty Liver. J Biol Chem (2012) 287(48):40732-44. doi: 10.1074/jbc.M112.399899

40. Simental-Mendía LE, Rodríguez-Morán M, Guerrero-Romero F. The Product of Fasting Glucose and Triglycerides as Surrogate for Identifying Insulin Resistance in Apparently Healthy Subjects. Metab Syndr Relat Disord (2008) 6 (4):299-304. doi: 10.1089/met.2008.0034

41. Guerrero-Romero F, Simental-Mendía LE, González-Ortiz M, MartínezAbundis E, Ramos-Zavala MG, Hernández-González SO, et al. The Product of Triglycerides and Glucose, a Simple Measure of Insulin Sensitivity. Comparison With the Euglycemic-Hyperinsulinemic Clamp. J Clin Endocrinol Metab (2010) 95(7):3347-51. doi: 10.1210/jc.2010-0288

42. Mirr M, Skrypnik D, Bogdański P, Owecki M. Newly Proposed Insulin Resistance Indexes Called Tyg-Nc and Tyg-Nhtr Show Efficacy in Diagnosing the Metabolic Syndrome. J Endocrinol Invest (2021) 44 (12):2831-43. doi: 10.1007/s40618-021-01608-2

43. Park K, Ahn CW, Lee SB, Kang S, Nam JS, Lee BK, et al. Elevated Tyg Index Predicts Progression of Coronary Artery Calcification. Diabetes Care (2019) 42(8):1569-73. doi: $10.2337 / \mathrm{dc} 18-1920$
44. Lee SB, Ahn CW, Lee BK, Kang S, Nam JS, You JH, et al. Association Between Triglyceride Glucose Index and Arterial Stiffness in Korean Adults. Cardiovasc Diabetol (2018) 17(1):41. doi: 10.1186/s12933-018-0692-1

45. Shi W, Xing L, Jing L, Tian Y, Liu S. Usefulness of Triglyceride-Glucose Index for Estimating Hyperuricemia Risk: Insights From a General Population. Postgrad Med (2019) 131(5):348-56. doi: 10.1080/00325481.2019.1624581

46. Cui N, Cui J, Sun J, Xu X, Aslam B, Bai L, et al. Triglycerides and Total Cholesterol Concentrations in Association With Hyperuricemia in Chinese Adults in Qingdao, China. Risk Manag Healthc Policy (2020) 13:165-73. doi: 10.2147/RMHP.S243381

Conflict of Interest: The authors declare that the research was conducted in the absence of any commercial or financial relationships that could be construed as a potential conflict of interest.

Publisher's Note: All claims expressed in this article are solely those of the authors and do not necessarily represent those of their affiliated organizations, or those of the publisher, the editors and the reviewers. Any product that may be evaluated in this article, or claim that may be made by its manufacturer, is not guaranteed or endorsed by the publisher.

Copyright (c) 2022 Kahaer, Zhang, Chen, Liang, He, Chen, Li, Tian, Hu and Sun. This is an open-access article distributed under the terms of the Creative Commons Attribution License (CC BY). The use, distribution or reproduction in other forums is permitted, provided the original author(s) and the copyright owner(s) are credited and that the original publication in this journal is cited, in accordance with accepted academic practice. No use, distribution or reproduction is permitted which does not comply with these terms. 